\title{
iTRAQ Based Protein Profile Analysis Revealed Key Proteins Involved in Regulation of Drought- Tolerance During Seed Germination in Adzuki Bean
}

\section{Xuesong Han}

Hubei Academy of Agricultural Sciences

\section{Fangwen Yang}

Shanghai Agrobiological Gene Center

\section{Yongguo Zhao}

Guangdong University of Petrochemical Technology

\section{Hongwei Chen}

Hubei Academy of Agricultural Sciences

\section{Zhenghuang Wan}

Hubei Academy of Agricultural Sciences

\section{Li Li}

Hubei Academy of Agricultural Sciences

\section{Longqing Sun}

Hubei Academy of Agricultural Sciences

\section{Liangjun Liu}

Hubei Academy of Agricultural Sciences

chunhai Jiao

Hubei Academy of Agricultural Sciences

Changyan Liu

Hubei Academy of Agricultural Sciences

Aihua Sha ( $\nabla$ aihuasha@yangtzeu.edu.cn )

Yangtze University

\section{Research Article}

Keywords: Ectopic expression, POD, PVX-LIC, V-ATPase, Water loss

Posted Date: August 5th, 2021

DOI: https://doi.org/10.21203/rs.3.rs-762775/v1 
License: (c) (i) This work is licensed under a Creative Commons Attribution 4.0 International License. Read Full License

Version of Record: A version of this preprint was published at Scientific Reports on December 1st, 2021. See the published version at https://doi.org/10.1038/s41598-021-03178-y. 


\section{Abstract}

Adzuki bean is famous as its high-quality protein, fiber, vitamins, minerals as well as rich bioactive substances. However, it is vulnerable to drought at the germination stage. Up to date, little information is available about the genetic controls of drought tolerance during seed germination in adzuki bean. In this study, differential expression proteins $\triangle \mathrm{DEP} \triangle \otimes$ were identified based on iTRAQ technology during seed germination between the drought-tolerant variety 17235 and drought-sensitive variety 17033 in adzuki bean. A total of 2834 proteins were identified in the two adzuki bean in the germinating seeds. Eightyseven and eighty DEPs were increased and decreased accumulation in variety 17235 compared to 17033 under drought, respectively. Meanwhile, 132/205 and 144/123 DEPs were up- or down-regulated in 17235 and 17033 under drought compared to the control, respectively. GO, KEGG, and PPI analysis revealed that the proteins related to carbohydrate metabolism and energy production showed abundantly increase in response to drought stresses. Ectopic overexpression of one candidate encoding V-ATPase in tobacco enhanced the drought tolerance of plants. The results provide valuable insights into adzuki bean response to drought stress, and the DEPs might be applied to develop drought tolerant adzuki bean in breeding programs.

\section{Introduction}

Drought is one of most devastating environmental stress that decreases crop productivity ${ }^{1}$. The occurrence of drought will be more frequent as the global temperature is increasing and the fresh water is lacking $^{2}$. Drought stress impact more seriously on germinating seeds and seedling development phases in most crops, which results in the delaying of seed germination and reduction of the germination rate at a very early developmental stage ${ }^{3}$. The establishment, growth, and productivity of crop is required for a high rate and uniformity of germination under drought, thus, understanding the genetics involved in seed germination under drought is helpful for further increasing yield potential ${ }^{3}$.

Seed germination is accomplished by a well-orchestrated series of events such as phytohormones and other small molecules mediated interactions with the environment, which signal a suitable environment for germination to ensure plant survival ${ }^{4}$. Specifically, ABA and GAs are considered to be indispensable for seed germination, and their dynamic equilibrium is central to the control of seed dormancy and germination ${ }^{5}$. Drought stress can delay or prevent the seed germination through reducing water availability, changing the mobilization of stored reserves, and affecting the structural organization of proteins ${ }^{6}$. To adapt to drought stress, plants developed the concerted mechanisms such as mechanoreceptors, ion transport channels, and secondary signal molecules to maintain ion homeostasis as well as cascades of gene activations for hormonal metabolism, signal transduction pathways, and stress responses ${ }^{7}$.

Adzuki bean (Vigna angularis $\mathrm{L}$ ) belongs to the Vigna of Leguminosae ${ }^{8}$. It is the second-most important legume crop behind soybean and is widely grown in East Asian countries such as China, Japan, and 
Korea $^{9}$. Adzuki bean is favored by Asian consumers due to its high-quality protein, fiber, vitamins, minerals as well as rich bioactive substances ${ }^{10,11}$. Although Adzuki bean was often planted in dry land as a paddy crop $^{12}$, it is vulnerable to drought at the germination stage ${ }^{13}$. Therefore, it is significant to improve its drought resistance to obtain the ideal productivity in arid environments. Understanding the genetics and exploiting key genes controlling drought tolerance at the germination stage of adzuki bean will be beneficial to create drought tolerant adzuki bean.

Recently, a number of genes, QTLs, or proteins in germinating seed responding to drought were identified in different crops. A genome-wide association scan (GWAS) analysis identified 338 single nucleotide polymorphisms (SNPs) were associated with seed germination-related traits under drought in barley ${ }^{3}$. Ten Quantitative trait loci (QTL) were detected to contribute to germination or early seedling drought tolerance in the interspecific cross Setaria italic $\times$ Setaria viridis ${ }^{14}$. Thirty-nine QTLs were identified in response to drought stress during seed germination in Brassica napus, in which 256 candidate genes were obtained by co-linear analysis between genetic and physical maps ${ }^{15}$. A total of 1200 proteins were detected to differentially accumulate under drought stress in maize by proteomics analysis ${ }^{16}$.

Up to date, little information is available about the genetic controls of drought tolerance during seed germination in adzuki bean. Several bZIP genes were reported in response to drought and salt stress in Vigna radiata and Vigna angularis by whole-genome sequencing and quantitative real-time PCR analysis ${ }^{17}$. We previously identified 82 differentially expressed genes (DEGs) in the germinating seeds between drought tolerant and drought sensitive variety of adzuki bean under drought stress by PacBio SMRT and Illumina Sequencing ${ }^{18}$.

In this study, the proteomics approach was adopted to further identify the differentially accumulated proteins (DEPs) in germinating seed of Adzuki bean by comparing the drought-tolerant variety (17235) and drought-tolerant variety (17033). The proteins related to carbohydrate metabolism and energy production showed abundantly increase in response to drought stresses, which were the potential candidates controlling drought resistance in germinating seeds of adzuki bean. The findings are helpful to understand the genetic controls of drought-stress response during seed germination, and provide foundation for breeding drought-tolerant adzuki cultivars with genetic engineering approach.

\section{Result}

\section{Quantitative proteomic analysis}

The previously identified drought-tolerant and drought sensitive varieties 17235 and $17033^{13}$, were used to conduct the proteomic analysis in this study. The seeds of 17235 and 17033 were treated with mannitol (MA) solution (designated to $17235 \mathrm{~T}$ and 17033T, respectively) or deionized water as control (designated to $17235 \mathrm{CK}$ and $17033 \mathrm{CK}$, respectively). The seeds with $24 \mathrm{~h}$ treatment were collected to analyze protein profiles based on the iTRAQ technology. A total of 179,175 spectra were utilized after 
eliminating the low-scoring spectra. Ultimately, 14,563 peptides, 10,764 unique peptides, and 2,834 protein groups were identified. The molecular weights of $93.33 \%$ of the identified proteins were up to 10 $\mathrm{kDa}$. The mass deviation of all identified peptides was mainly distributed within $10 \mathrm{ppm}$ with the ideal score of MASCOT. The score of about $80 \%$ of the peptides are beyond 20 , and the abundance ratio of most proteins in the two groups of equal-labeled samples is close to 1 (Table S1, S2).

\section{Different expression of identified proteins}

Drought-responsive proteins were identified based on a comparison of protein abundance between control and MA-treated samples using the iTRAQ data. A fold change ratio $>1.20$ or $<0.83(p<0.05)$ was used to identify differentially expressed proteins (DEPs) between the drought-treated and control seeds.

To investigate what proteins might determine the tolerant difference of the two varieties, we firstly focus on the DEPs in comparison of $17235 \mathrm{~T}$ and 17033T. A total of 167 DEPs were identified between them, of which, 87 were up-regulated and 80 down-regulated, respectively (Fig. 1A, Table S3). As the control, 30 DEPs were identified between 17235CK and 17033CK (Fig. 1A, Table S3). Notably, five DEPs were identified both in the comparisons of 17235T-17033T and 17235CK-17033CK (Fig. 1B, Table S3), implying these DEPs might be variety-specific proteins. To investigate drought-responsive proteins in different variety, we analyzed the DEPs in the comparisons of 17235T-17235CK and 17033T-17033CK. A total of 337 and 267 DEPs were identified in the comparisons of 17235T-17235CK and 17033T-17033CK, respectively. Among them, 132/205 and 144/123 DEPs were up- or down-regulated in 17235T and 17033T compared to the control, respectively (Fig. 1A, Table S3).

\section{Functional annotation of drought-responsive proteins}

The function information of all DEPs were obtained by searching against the UniProt-GOA database, which were assigned to three categories based on $\mathrm{GO}$ annotation, that is, cellular compartment, biological process, and molecular function. The DEPs in comparison of 17235T-17033T belonged to 111 biological processes, 101 cellular compartments, and 125 molecular functions, respectively (Table S4). In terms of biological process, metabolic process and cellular process were the major groups. Catalytic activity and binding were the top two major molecular functional groups. Cell and cell part were the top two cellular compartments. "structural molecule activity", "structural constituent of ribosome" were enriched by DEPs in the category of molecular function, indicating that structural molecule activity play a major role in regulating drought tolerance between different varieties; In the category of cellular component, the GO terms related with several cytosolic proteins were detected, such as "cytosolic large ribosomal subunit", "cytosolic part", "cytosol", "cytosolic ribosome" (Fig. 2). These cytosolic proteins may be involved in the regulation of seed permeation to regulate drought tolerance in germinating seeds.

KEGG analysis indicated that DEPs in comparison of $17235 \mathrm{~T}-17033 \mathrm{~T}$ assigned to $110 \mathrm{KEGG}$ pathways. Among them, four pathway categories were significantly enriched with the $p$-value lower than 0.05 from. The four pathways were Ribosome, Phagosome, Fructose and mannose metabolism, and Insulin 
signaling pathway, implying that there were correlated to the different drought tolerance between 17235 and 17033 (Table S5).

\section{Protein-Protein Interaction among DEPs}

To predict the relationship among all these identified DEPs in adzuki bean, a protein-protein interaction (PPI) networks were generated using the web-tool STRING 9.1. A total of 167 DEPs represented by 99 unique proteins from adzuki bean were shown in the PPI network (Fig. 3, Table S6,).

Four functional modules forming tightly-connected clusters were illuminated in the network. Nodes in different colors belong to the major metabolic pathways. In Module RNA-binding Proteins, a large number of ribosomal proteins are closely linked to the RNA-binding proteins and transport RNA. It indicated that the ribosome pathway gene plays a major role in the early germination of Adzuki bean seeds under drought stress. Plant cytoskeleton related proteins connected together in Module Cellular structure,This also shows that the cell structure plays a vital role in seed germination under drought stress. Module Energy metabolism included multiple enzymes involved in the TCA cycle, glycolysis, amino acid metabolism related proteins and nitrogen metabolism. These linked proteins indicated that a synergistic system for carbon and nitrogen metabolism may play important roles in drought response. The module ROS regulation distribution is relatively scattered, indicating that ROS is produced in different metabolic processes, but it is related to energy metabolism, which also shows that the process of energy metabolism is closely related to the production and regulation of ROS.

\section{qRT-PCR assay}

To investigate transcription patterns, 11 identified proteins by iTRAQ underwent qRT-PCR analyses. Some of these DEPs have been reported to be involved in stress response,other proteins maybe related to stress response. The fold changes of protein are provided in Table S3 and qRT-PCR primers for analysis of mRNA expression are provided in Table S7. A comparison of the qRT-PCR data for MA-treated and control samples indicated that 11 DEPs (A0A0L9VAK7, A0A0L9TF45, A0A0L9TJ92, A0A0L9TQX2, A0A0L9U7B9, A0A0L9UWB5, A0A0L9V113, AOA0L9V7V3, A0A0L9VCX3, A0A0L9VM15, A0A0L9VS04) had the consistent change fold between transcript and protein. The results suggested that the changes of transcript and protein level were similar for most of the analyzed DEPs (Fig. 4).

\section{Functional verification of DEP}

V-ATPase (Vacuolar $\mathrm{H}^{+}$-ATPases, VHA) regulates the ion balance of cell in plants by pumping $\mathrm{H}^{+}$from the cytosol into the vacuole. V-ATPase was reported to survive plant cell by enhancing its activity under salinity and drought ${ }^{19,20}$. One DEP, AOAOL9TJ92, encoded V-ATPase and indicated the consistent expression at transcriptional and translational level in this study, which was up-regulated in 17235T compared to $17033 \mathrm{~T}$. To verify whether this protein can improve the drought tolerant in plant, the fulllength cDNA of VaVHA-c was amplified from Adzuki seeds and was ectopic overexpressed in Nicotiana benthamiana by the PVX virus vector. The ectopic overexpression of VaVHA-c obviously enhanced the 
drought tolerance in tobacco. The plants with ectopic overexpression of VaVHA-c grew normally, however, the control or plants inoculated with empty vector wilted after $15 \mathrm{~d}$ drought treatment (Fig. 5A). The expression of $V a V H A-c$ can be detected only in the ectopic overexpression of VaVHA-c plants by RT-PCR (Fig. 5B), implying that the enhanced drought tolerance was due to the overexpression of VaVHA-C. Measurements of physiological parameters indicated that the activity of peroxidase (POD) was increased, and water loss was reduced in the leaves of plants with overexpressed VaVHA-c under drought (Fig. 6).

\section{Discussion}

The germination efficiency determines the seedling establishment and the proper development of mature plants. Germination is a complex process involving events associated with the transition of a quiescent dry seed to a metabolically active state ${ }^{21,22}$. In the study, a number of DEPs were detected between the drought tolerant and drought sensitive varieties (Fig. 1), and four functional modules Energy metabolism, Cellular structure, ROS regulation, RNA-binding Proteins were formed tightly-connected clusters in PPI network in this study (Fig. 3), implying that DEPs involved in these processes might played the key roles in regulatory of germination under stress.

The cytoskeleton-associated proteins have been shown to be key regulatory molecules in mediating cytoskeleton reorganization in response to multiple environmental signals, such as light, salt, drought and biotic stimuli ${ }^{23}$. Reorganization of cellular structure Actin is a central component of the cytoskeleton ${ }^{3}$. Actin and the microtubule network are regulated by many factors such as $\mathrm{EF} 1 \mathrm{a}, \mathrm{Ca}^{2+} / \mathrm{CaM}$, tubulin cofactors (TBCC) ${ }^{24-27}$. OsADF3-heterologous transgenic Arabidopsis increased drought stress tolerance and up-regulated many downstream drought-tolerant responsive genes ${ }^{28}$. Overexpression of LreEF1A4 improved seed germination rate under drought $\operatorname{stress}^{29}$. In this study, we detected several up-regulated DEPs related to cell structure such as A0A0L9TKB7(Tubulin alpha chain), A0A0S3RAJ6 (Tubulin beta chain), AOAOL9TDG2 (actin family), AOAOS3RXP8 (TBCC domain-containing protein 1), A0A0L9T825 (Tcomplex protein 1 subunit gamma), A0A0S3RK85 (T-complex protein 1 subunit eta) (Fig. 7 and Table S8) under drought stress in 17235 compared to 17033.These proteins are very likely to provide a good structural basis for seed germination under drought stress. In addition, the cell wall-related proteins NC_030646.1.428.73, A0A0S3SNK4 (Dirigent protein) $\square$ NC_030641.1.847.6 (epidermis-specific secreted glycoprotein EP1)\A0A0S3SEG8 (expansin) (Fig. 7 and Table S8) were contrary to previous reports ${ }^{30-32}$, which may be the specific metabolic activity of adzuki bean under drought conditions.

Energy materials including carbohydrates, proteins and lipids in seeds are utilized as nutrients and energy sources at the germination stage. Starch and proteins are mobilized via the activation of corresponding amylases and proteases ${ }^{33}$. The carbohydrate can be utilized more easily in metabolism due to its simple molecular structure ${ }^{34}$. The enhanced carbohydrate metabolism would accelerate reserve hydrolysis and impair protein biosynthesis during seed germination ${ }^{35}$. In this study, quantities of DEPs were detected in the pathways related with energy and carbohydrate metabolism, such as alpha-1,4 glucan phosphorylase, 
pyrophosphate-fructose 6-phosphate 1-phosphotransferase, sucrose synthase. Most of these DEPs were up-regulated, and five seed storage proteins A0AOS3SMN4, AOAOS3SMS9, NC_030638.1.306.23, A4PI99, NC_030642.1.591.12 (Fig. 7 and Table S8) were significantly down-regulated in drought-tolerant variety 17235 than sensitive variety 17033 under drought stress, implying that more energy provided might result in the drought-tolerance of 17235 under drought stress.

In addition, Storage proteins must be degraded to sustain embryo growth and development until an autotrophic growth is reached. Several protease families are involved in the germination process ${ }^{36}$. There are 9 proteases up-regulated and 8 protease inhibitors are down-regulated in 17235 than 17033 under drought (Fig. 7 and Table S8), suggesting that the seeds of drought-tolerant varieties use a large amount of proteases to decompose storage proteins to provide energy for their germination and promote seed germination, whereas the sensitive variety seeds decompose more slowly due to protease inhibitors, and inhibit seed germination.

ROS can participate in endosperm weakening during germination through cell wall loosening, but uncontrolled ROS production can lead to oxidative stress and cellular damage, resulting in seed deterioration, preventing germination and early seedling development ${ }^{37,38}$. Some antioxidants such as superoxide enzyme (SOD), peroxidase (POD), glutathione-related enzyme and dehydrogenase was produced to decrease the cellular damage caused by ROS in plants ${ }^{39}$. In this study, DEPS NC_030637.1.1703.10, AOAOL9U8C9, NC_030637.1.740.3, and A0A0S3SSI5 (Fig. 7 and Table S7), which are involved in regulating ROS homeostasis ${ }^{40-42}$, were detected, suggesting maintenance of ROS homeostasis is important for drought tolerance in Adzuki seed germination.

V-ATPase was reported to participated in stress response ${ }^{19,20}$. In this study, we validated that one DEP encoding VaVHA-c could enhance the drought tolerance of plants by overexpressing it in Nicotiana benthamiana (Fig. 5). Furthermore, the POD activity was increased, and the water loss was reduced in the leaves of overexpressed tobacco plants compared to the controls under drought stress (Fig. 6).

\section{Materials And Methods}

\section{Plant growth conditions and treatments}

The seeds of s17235 and s17033 were germinated in mannitol (7.5\% concentration) and deionized water for $24 \mathrm{~h}$, as described by Zhu et al ${ }^{13}$. The samples were collected in $24 \mathrm{~h}$, immediately frozen and stored in liquid nitrogen for protein and RNA extraction. Three biological replicates were conducted for each treatment.

\section{Protein extraction, digestion and ITRAQ labelling}

Total proteins were extracted using the cold acetone method. Samples were ground to power in liquid nitrogen, then dissolved in $2 \mathrm{~mL}$ lysis buffer (8 M urea, $2 \%$ SDS, $1 \times$ Protease Inhibitor Cocktail (Roche Ltd. Basel, Switzerland), followed by sonication on ice for $30 \mathrm{~min}$ and centrifugation at $13000 \mathrm{rpm}$ for $30 \mathrm{~min}$ 
at $4^{\circ} \mathrm{C}$. The supernatant was transferred to a fresh tube. For each sample, proteins were precipitated with ice-cold acetone at $-20^{\circ} \mathrm{C}$ overnight. The precipitations were cleaned with acetone three times and redissolved in $8 \mathrm{M}$ Urea by sonication on ice. Protein quality was examined with SDS-PAGE.

BCA protein assay was used to determine the protein concentration of the supernatant. $100 \mu \mathrm{g}$ protein per condition was transferred into a new tube and adjusted to a final volume of $100 \mu \mathrm{L}$ with $8 \mathrm{M}$ Urea. $11 \mu \mathrm{L}$ of $1 \mathrm{M}$ DTT (DL-Dithiothreitol) was added and samples were incubated at $37^{\circ} \mathrm{C}$ for 1 hour. Then $120 \mu \mathrm{L}$ of the $55 \mathrm{mM}$ iodoacetamide was added to the sample and incubated for 20 minutes protected from light at room temperature.

For each sample, proteins were precipitated with ice-cold acetone, then re-dissolved in $100 \mu \mathrm{L}$ TEAB. Proteins were then tryptic digested with sequence-grade modified trypsin (Promega, Madison, $\mathrm{WI}$ ) at $37^{\circ} \mathrm{C}$ overnight. The resultant peptide mixture was labeled with iTRAQ tags 113 through 118 . The labeled samples were combined and dried in vacuum.

\section{Strong cation exchange (SCX) fractionation and liquid chromatography-tandem mass spectrometry (LC- MS/MS) analysis}

The combined labeled samples were subjected to the SCX fractionation column connected with a high performance liquid chromatography (HPLC) system. The peptide mixture was re-dissolved in the buffer A (buffer A: $20 \mathrm{mM}$ ammonium formate in water, $\mathrm{pH} 10.0$, adjusted with ammonium hydroxide), and then fractionated by high pH separation using Ultimate 3000 system (Thermo Fisher scientific, MA, USA) connected to a reverse phase column (XBridge C18 column, 4.6mm x $250 \mathrm{~mm}$, 5 $\mu \mathrm{m}$, (Waters Corporation, MA, USA). High pH separation was performed using a linear gradient starting from $5 \% \mathrm{~B}$ to $45 \% \mathrm{~B}$ in 40 min (B: $20 \mathrm{mM}$ ammonium formate in $80 \% \mathrm{ACN}, \mathrm{pH} 10.0$, adjusted with ammonium hydroxide). The column was re-equilibrated at initial conditions for $15 \mathrm{~min}$. The column flow rate was maintained at $1 \mathrm{~mL} / \mathrm{min}$ and column temperature was maintained at $30^{\circ} \mathrm{C}$. Twelve fractions were collected; each fraction was dried in a vacuum concentrator for the next step.

Peptide fractions were resuspended with $30 \mu$ solvent C respectively (C: water with $0.1 \%$ formic acid; D: ACN with $0.1 \%$ formic acid), separated by nanoLC and analyzed by on-line electrospray tandem mass spectrometry. The experiments were performed on an Easy-nLC 1000 system (Thermo Fisher Scientific, MA, USA) connected to a Orbitrap Fusion Tribrid mass spectrometer (Thermo Fisher Scientific, MA, USA) equipped with an online nano-electrospray ion source. $10 \mu$ peptide sample was loaded onto the trap

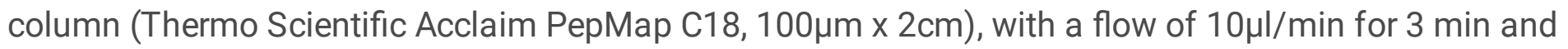
subsequently separated on the analytical column (Acclaim PepMap C18,75 $\mathrm{m} \times 15 \mathrm{~cm}$ ) with a linear gradient, from $3 \% \mathrm{D}$ to $32 \% \mathrm{D}$ in $120 \mathrm{~min}$. The column was re-equilibrated at initial conditions for $10 \mathrm{~min}$. The column flow rate was maintained at $300 \mathrm{~nL} / \mathrm{min}$. The electrospray voltage of $2 \mathrm{kV}$ versus the inlet of the mass spectrometer was used.

The fusion mass spectrometer was operated in the data-dependent mode to switch automatically between MS and MS/MS acquisition. Survey full-scan MS spectra (m/z 350-1550) were acquired with a 
mass resolution of $120 \mathrm{~K}$, followed by sequential high energy collisional dissociation (HCD) MS/MS scans with a resolution of $30 \mathrm{~K}$. The isolation window was set as $1.6 \mathrm{Da}$. The AGC target was set as 400000. MS/MS fixed first mass was set at 110. In all cases, one microscan was recorded using dynamic exclusion of 45 seconds.

\section{Database search}

The mass spectrometry data were transformed into MGF files with Proteome Discovery 1.2 (Thermo, Pittsburgh, PA, USA) and analyzed using Mascot search engine (Matrix Science, London, UK; version 2.3.2). Mascot database was set up for protein identification using Vigna angularis $L$ reference transcriptome or Vigna angularis $L$ database in NCBInr/SwissProt/Uniprot/IPI, and the PacBio SMRT and Illumina Sequencing data published by Zhu et $\mathrm{al}^{18}$. Mascot was searched with a fragment ion mass tolerance of $0.050 \mathrm{Da}$ and a parent ion tolerance of 10.0 PPM.

\section{Protein identification and quantification}

The Mascot search results were averaged using medians and quantified. Proteins with fold change in a comparison $>1.2$ or $<0.83$ and unadjusted significance level $p<0.05$ were considered differentially expressed.

\section{GO Enrichment analysis}

Gene Ontology (GO) is an international standardized gene functional classification system which offers a dynamic-updated controlled vocabulary and a strictly defined concept to comprehensively describe properties of genes and their products in any organism. GO has three ontologies: molecular function, cellular component and biological process. The basic unit of GO is GO-term. Each GO-term belongs to a type of ontology.

GO enrichment analysis provides all GO terms that significantly enriched in DEPs comparing to the genome background, and filter the DEGs that correspond to biological functions. Firstly, all DEPs were mapped to GO terms in the Gene Ontology database (http://www.geneontology.org/), gene numbers were calculated for every term, significantly enriched GO terms in DEGs comparing to the genome background were defined by hypergeometric test. The calculated p-value was gone through FDR Correction, taking FDR $\leq 0.05$ as a threshold. GO terms meeting this condition were defined as significantly enriched GO terms in DEPs. This analysis was able to recognize the main biological functions that DEPs exercise.

\section{Pathway enrichment analysis}

Pathway-based analysis was conducted by blasting against for KEGG database (https://www.kegg.jp/kegg/pathway.html). Pathway enrichment analysis identified significantly enriched metabolic pathways or signal transduction pathways in DEPs comparing with the whole genome background. The calculated p-value was gone through FDR Correction, taking FDR $\leq 0.05$ as a threshold. Pathways meeting this condition were defined as significantly enriched pathways in DEPs. 


\section{RNA extraction and qRT-PCR}

Total RNA was extracted using the TRIZOL reagent (Invitrogen, Carlsbad, CA, USA), and then treated with RNasefree DNase (Invitrogen, Gaithersburg, MD, USA). The purified RNA was reverse transcribed using the RevertAid $^{\text {TM }}$ First Strand cDNA Synthesis Kit (Thermo Fisher Scientific) according to the manufacturer's protocol. The qRT-PCR reactions were performed in CFX96 ${ }^{\text {TM }}$ Real-Time PCR Detection System (Bio-Rad, USA). The gene specific primers were listed in additional Table S5 Each reaction was conducted in $10 \mu \mathrm{l}$ mixture containing $5 \mu$ l of SYBR green (SYBR@ Premix Ex Taq ${ }^{\text {TM }}$ (TliRNaseH Plus), TAKARA, Japan), $0.3 \mu l$ forward and reverse primers $(10 \mu \mathrm{M})$, respectively, $2 \mu \mathrm{l}$ cDNA template, and $2.4 \mu \mathrm{ldH}_{2} \mathrm{O}$. The reactions for each gene were conducted in triplicate with the thermal cycling conditions as follows: $95^{\circ} \mathrm{C}$ for $30 \mathrm{~s}$, followed by 40 cycles of $95^{\circ} \mathrm{C}$ for $5 \mathrm{~s}$ and $57^{\circ} \mathrm{C}$ for $30 \mathrm{~s}$. The primer specificity was confirmed by melting curve analysis. Relative expression levels of the genes were calculated using the $2^{-\triangle \Delta C T}$ method ${ }^{43}$.

\section{Ectopic expression of VaVHA-c and RT-PCR}

Four weeks old $N$. benthamiana plants grown in a growth room $\left(24^{\circ} \mathrm{C}, 16 \mathrm{~h} / 8 \mathrm{~h} \mathrm{light} /\right.$ dark, $100 \mu \mathrm{M} \mathrm{m}^{-2} \mathrm{~s}^{-}$

${ }^{1}$ white light) were used for VaVHA-c ectopic expression. The coding sequence (CDS) region of VaVHA-c was amplified with gene specific primers and was inserted into PVX-LIC vector as described by Zhao et $\mathrm{al}^{44}$, and confirmed by sequencing. The resultant construct VaVHA-C-PVX-LIC was introduced into Agrobacterium tumefaciens GV3101 via the freeze-thaw method, and then introduced into tobacco (Nicotiana benthimiana) by infiltration method ${ }^{44}$ (Zhao et al. 2016). The empty vector PVX-LIC was introduced into tobacco as negative control. The experiment was performed three times with at least 5 plants for each construct. After $7 \mathrm{~d}$ of infiltration, the leaves were harvested for RNA extraction and RTPCR analysis, and the plants were treated by withholding water. The phenotype of plants was photographed at $15 \mathrm{~d}$ after water withholding. Gene-specific primers were used for RT-PCR and actin was used to normalize the reaction as described by Sha et $\mathrm{al}^{43}$.

\section{Physiological Parameter Measurements and Statistical Analysis}

The activity of superoxide dismutase (SOD), peroxidase (POD), content of proline and malondialdehyde (MDA), and water Loss were analyzed as described by Zhou et al ${ }^{45}$. Data analysis were conducted by Microsoft Excel 2016 and the software of SPSS 16.0. The significance was analyzed by One-way ANOVA test. Tukey multiple comparison test was used to compared differences at 0.05 significance level.

\section{Declarations}

\section{Acknowlegments}

This work was supported by grants from National Key R\&D Program of China ه2019YFD1001305/2019YFD1001300囚, China Agriculture Research System of MOF and MARA-Food Legumes (CARS-08-G13), Major Program of Hubei Province (2018ABA090). 


\section{Author Contributions}

XH conducted the ITRAQ analysis, FY prepared the figures, HC and ZW performed data analysis, LL performed the qPCR analysis, $L S$ and $L J L$ prepare the germinating seeds, $C J$ reviewed the manuscript, $C L$ and $A S$ designed the experiments, $\mathrm{XH}, \mathrm{FY}$, and $\mathrm{YZ}$ wrote the manuscript. All authors read and approved the final manuscript.

\section{Competing Interest}

The authors declare no competing interest.

\section{References}

1. Lambers, H., Chapin, F.S., Pons, T.L. Plant water relations. Plant physiological ecology 163223(Springer, New York, NY, 2008).

2. Xie, H., Yang, D.H., Yao, H., Bai, G., Zhang, Y.H., Xiao, B.G. iTRAQ-based quantitative proteomic analysis reveals proteomic changes in leaves of cultivated tobacco (Nicotiana tabacum) in response to drought stress. Biochem Biophys Res Commun 469, 768-775 (2016).

3. Thabet, S.G., Moursi, Y.S., Karam, M.A., Graner, A., Alqudah, A.M. Genetic basis of drought tolerance during seed germination in barley. PloS one 13(11), e0206682 (2018).

4. Yang, R., et al. The mechanism underlying fast germination of tomato cultivar LA2711. Plant Sci 238, 241-250 (2015).

5. Feurtado, J.A., Kermode, A.R. Annual plant reviews $\|$ a merging of paths: abscisic acid and hormonal cross-talk in the control of seed dormancy maintenance and alleviation. https//:10.1002/9781119312994, 176-223(2018).

6. Ibrahim, E.A. Seed priming to alleviate salinity stress in germinating seeds. J. Plant Physiol. 192, 3846(2016).

7. Zhu, J.K. Abiotic stress signaling and responses in plants. Cell 167, 313-324(2016)

8. Morris, J.B., Wang, M.L. Updated review of potential medicinal genetic resources in the USDA, ARS, PGRCU industrial and legume crop germplasm collections. Ind. Crops Prod. 123, 470-479 (2018).

9. Lestari, P., et al. Genome-wide single nucleotide poly-morphism discovery and validation in adzuki bean. Mol. Breed 33, 497-501(2014).

10. Kitano-Okada, T., et al. Anti-obesity role of adzuki bean extract containing polyphenols: In vivo and in vitro effects. J. Sci. Food Agric. 92, 2644-2651(2012).

11. Luo, J., Cai, W., Wu, T., Xu, B. Phytochemical distribution in hull and cotyledon of adzuki bean (Vigna angularis $\mathrm{L}$.) and mung bean (Vigna radiate L.), and their contribution to antioxidant, antiinflammatory and anti-diabetic activities. Food Chem. 201, 350-360 (2016). 
12. Chun, H.C., Jung, K.Y., Choi, Y.D., Sang, H.L., Hang, W.K. The growth and yield changes of foxtail millet (Setaria italic L.), proso millet (Panicum miliaceum L.), sorghum (Sorghum bicolor L.), adzuki bean (Vigna angularis L.), and sesame (Sesamum indicum L.) as affected by excessive soil-water. Korean J. Agric. Sci. 43, 547-559 (2016).

13. Zhu, Z., et al. Evaluation and screening of adzuki bean germplasm resources for drought tolerance during germination stage. J. South Agric. 50, 1183-1190 (2019).

14. Qie, L., et al. Mapping of Quantitative trait locus (QTLs) that contribute to germination and early seedling drought tolerance in the interspecific cross Setaria italica×Setaria viridis. PLoS one $\mathbf{9}$, e101868 (2014).

15. Gad, M., Chao, H., Li, H., Zhao, W., Lu, G., Li, M. QTL mapping for seed germination response to drought stress in Brassica napus. Front. Plant Sci. 11, 629970 (2021).

16. Liu, S., et al. Comparative proteomic and morpho-physiological analyses of maize wild-type Vp16 and mutant vp16 germinating seed responses to PEG-induced drought stress. Int. J. Mol. Sci. 20, 5586 (2019).

17. Wang, L., Zhu, J., Li, X., Wang, S., Wu, J. Salt and drought stress and ABA responses related to bZIP genes from V. radiate and V. angularis. Gene 651, 152-160 (2018).

18. Zhu, Z., et al. Characterization of drought-responsive transcriptome during seed germination in adzuki bean (Vigna angularis L.) by PacBio SMRT and Illumina sequencing. Front. Genet. 11, 996 (2020).

19. Dietz, K.J., et al. Significance of the V-type ATPase for the adaptation to stressful growth conditions and its regulation on the molecular and biochemical level. J. Exp. Bot. 52, 1969-1980 (2001).

20. Wang, L., He, X., Zhao, Y., Shen, Y., Huang, Z. Wheat vacuolar $\mathrm{H}^{+}$-ATPase subunit B cloning and its involvement in salt tolerance. Planta 234, 1-7 (2011).

21. Weitbrecht, K., Müller, K., Leubner-Metzger, G. First off the mark: early seed germination. J. Exp. Bot. 62(10), 3289-3309 (2011).

22. Yang, F.W., et al, Transcriptome profile analysis of two Vicia faba cultivars with contrasting salinity tolerance during seed germination. Scientific Reports 10, 7250 (2020)

23. Wang, X., Mao, T. Understanding the functions and mechanisms of plant cytoskeleton in response to environmental signals. Curr. Opin. Plant Biol. 52, 86-96 (2019).

24. Yoo, C.Y., et al. $\mathrm{A} \mathrm{Ca}^{2+} / \mathrm{CaM}$-regulated transcriptional switch modulates stomatal development in response to water deficit. Sci. Rep. 9(1), 12282 (2019).

25. Bunai, F., Ando, K., Ueno, H., Numata, O. Tetrahymena eukaryotic translation elongation factor $1 \mathrm{~A}$ (eEF1A) bundles filamentous actin through dimer formation. J. Biochem. 140(3), 393-399 (2006).

26. Stephan, A., et al. An essential quality control mechanism at the eukaryotic basal body prior to intraflagellar transport. Traffic 8(10), 1323-1330 (2007).

27. Fichtenbauer, D., Xu, X.M., Jackson, D., Kragler, F. The chaperonin CCT8 facilitates spread of tobamovirus infection. Plant Signal Behav. 7(3), 318-321 (2012). 
28. Huang, Y.C., Huang, W.L., Hong, C.Y., Lur, H.S., Chang, M.C. Comprehensive analysis of differentially expressed rice actin depolymerizing factor gene family and heterologous overexpression of OsADF3 confers Arabidopsis thaliana drought tolerance. Rice 5(1), 33 (2012).

29. Sun, D., et al. LreEF1A4, a Translation Elongation Factor from Lilium regale, is pivotal for cucumber mosaic virus and tobacco rattle virus infections and tolerance to salt and drought. Int. J. Mol. Sci. 21(6), 2083 (2020).

30. Guo, J., et al. A novel dirigent protein gene with highly stem-specific expression from sugarcane, response to drought, salt and oxidative stresses. Plant Cell Rep 31, 1801-1812 (2012).

31. van Engelen, F.A., et al. The carrot secreted glycoprotein gene EP1 is expressed in the epidermis and has sequence homology to Brassica S-locus glycoproteins. Plant J. 4, 855-862 (1993).

32. Marowa, P., Ding, A., Kong, Y. Expansins: roles in plant growth and potential applications in crop improvement. Plant Cell Rep. 35, 949-965 (2016).

33. Ma, Z., et al. Glyoxylate cycle and metabolism of organic acids in the scutellum of barley seeds during germination. Plant Sci. 248, 37-44 (2016).

34. Meis, S.J., Fehr, W.R., Schnebly, S.R. Seed source effect on field emergence of soybean lines with reduced phytate and raffinose saccharides. Crop Sci. 43, 1336-1339 (2003).

35. Aguirre, M., Kiegle, E., Leo, G., Ezquer, I. Carbohydrate reserves and seed development: an overview. Plant Reprod. 31, 263-290 (2018).

36. Diaz-Mendoza, M., Diaz, I., Martinez, M. Insights on the Proteases Involved in Barley and Wheat Grain Germination. Int. J. Mol. Sci. 20, 2087 (2019).

37. Müller, K., et al. In vivo cell wall loosening by hydroxyl radicals during cress seed germination and elongation growth. Plant Physiol. 150, 1855-1865 (2009).

38. Sattler, S.E., Gilliland, L.U., Magallanes-Lundback, M., Pollard, M., DellaPenna, D. Vitamin E is essential for seed longevity and for preventing lipid peroxidation during germination. Plant Cell 16, 1419-1432 (2004).

39. Fotopoulos, V., et al. Application of sodium nitroprusside results in distinct antioxidant gene expression patterns in leaves of mature and senescing Medicago truncatula plants. Protoplasma 251, 973-978 (2014).

40. Boyd, S.D., et al. The yeast copper chaperone for copper-zinc superoxide dismutase (CCS1) is a multifunctional chaperone promoting all levels of SOD1 maturation. J. Biol. Chem. 294, 1956-1966 (2019).

41. Garg, G., Neha, P. Plant transcription factors networking of pyrroline-5-carboxylate (p5c) enzyme under stress condition: a review. Plant Archives 19, 562-569 (2019).

42. Zhao, Y., Yu, H., Zhou, J.M., Smith, S.M., Li, J. Malate circulation: Linking chloroplast metabolism to mitochondrial ROS. Trends Plant Sci. 25, 5 (2020).

43. Sha, A.H., Gao, Z.L., Wu, H., Lin, D.Z., Zhang, Q.L., Chen, Y.H. Ectopic expression of soybean methionine synthase delays flowering time in transgenic tobacco plants. Biol Plant 59: $47-54$ (2015). 
44. Zhao, J., et al. An efficient Potato virus X-based microRNA silencing in Nicotiana benthamiana. Sci. Rep. 6, 20573 (2016).

45. Zhou, Z., Wang, J., Zhang, S., Yu, Q., Lan, H. Investigation of the nature of CgCDPK and CgbHLH001 interaction and the function of bHLH transcription factor in stress tolerance in Chenopodium glaucum. Front. Plant Sci. 11, 603298 (2021).

\section{Figures}

A

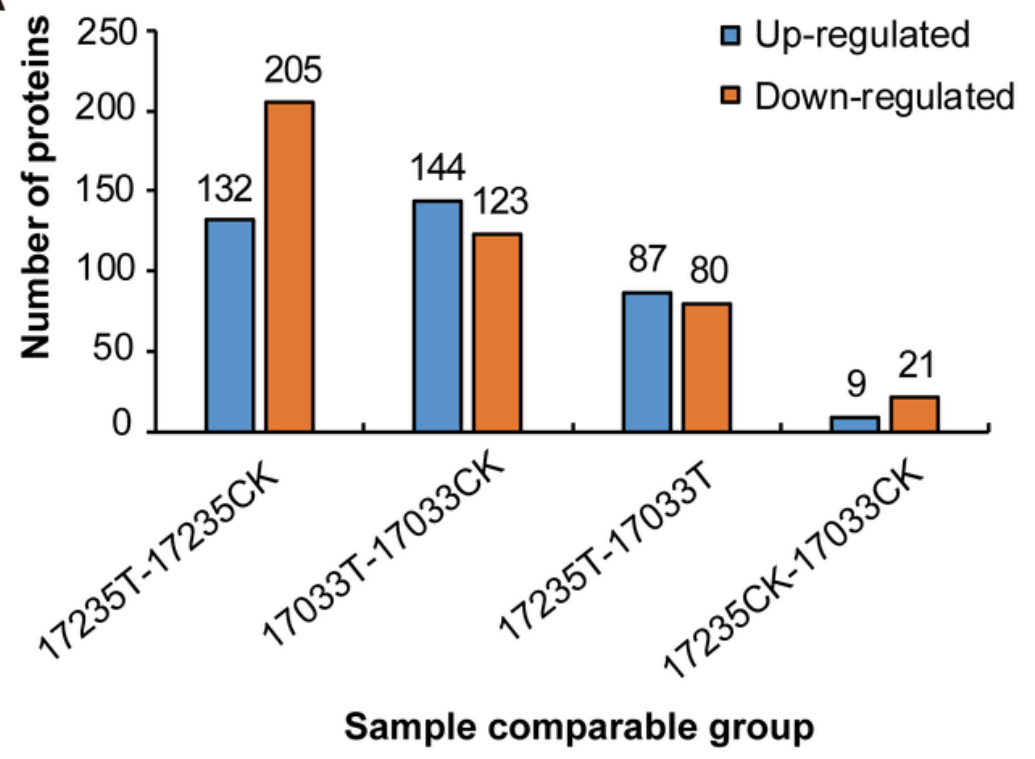

B

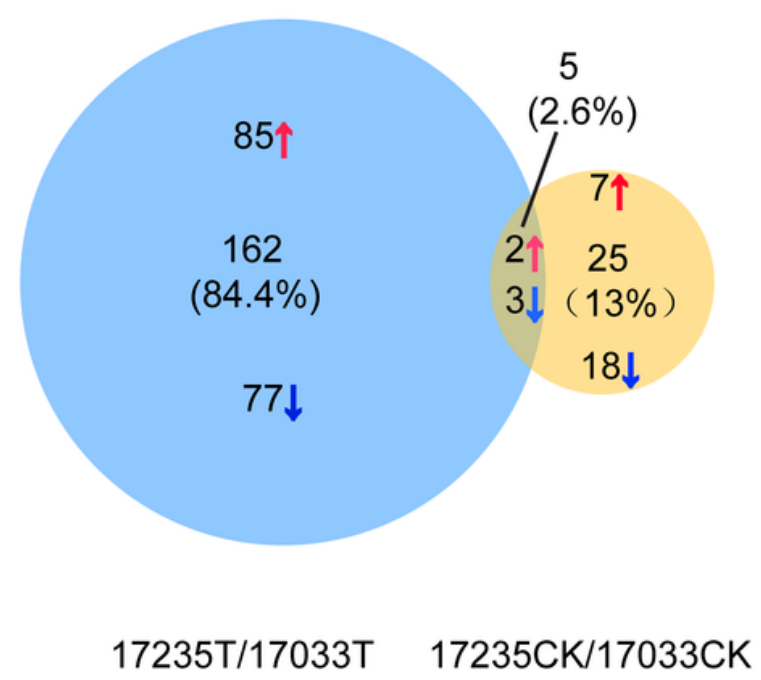

Figure 1

Quantitative and Venn analysis of the proteome of two Adzuki bean under different treatments. (A) Quantitative analysis of the proteome between the MA treated and control samples, (B) Venn analysis of two Adzuki bean under different treatments. 


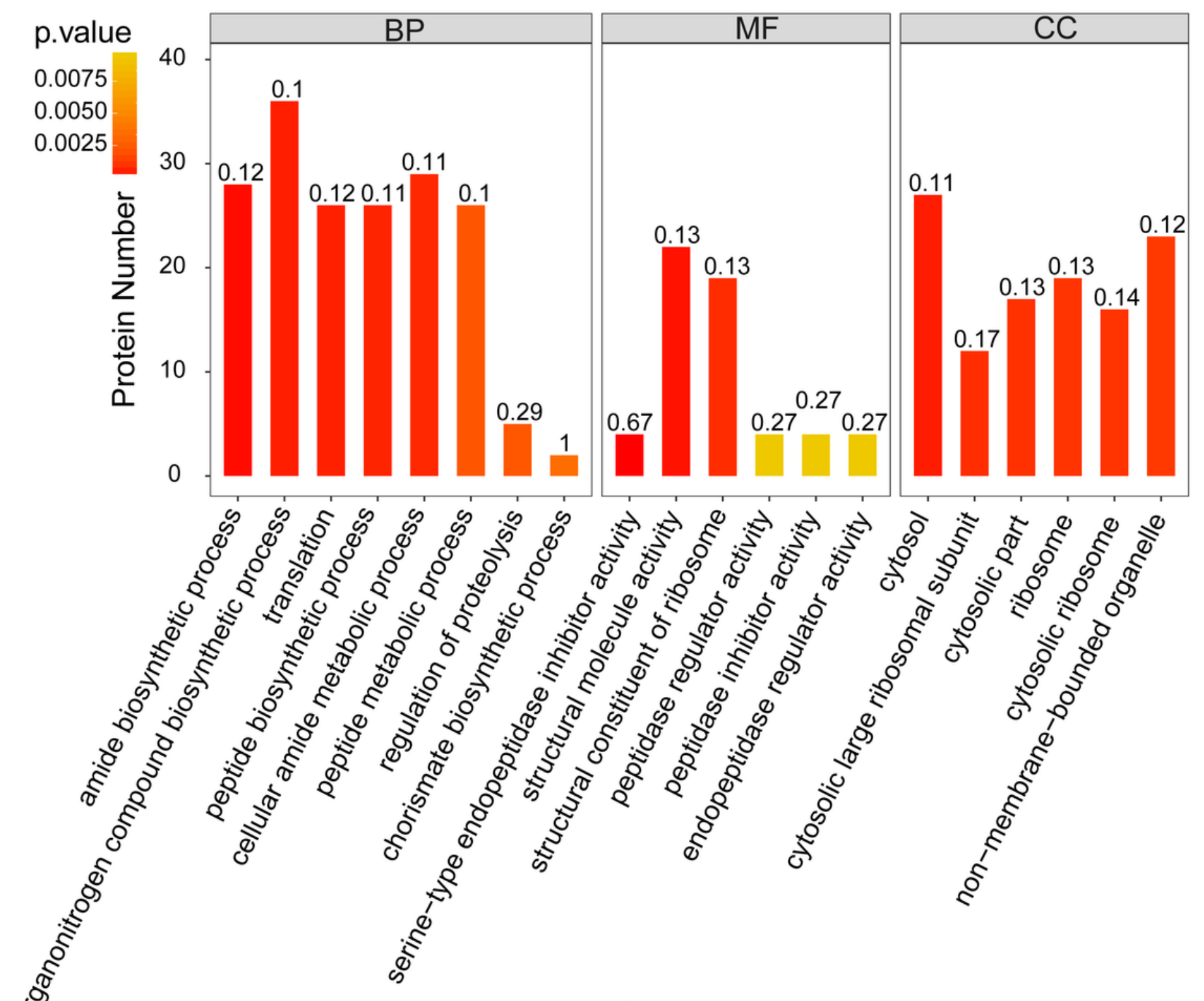

Enriched GO Terms (Top 20)

\section{Figure 2}

GO enrichment annotation of differentially expressed proteins between 17235 and 17033 under drought stress. The abscissa in the figure represents the enriched GO functional classification, BP (Biological Process,), MF (Molecular Function) and CC (Cellular Component) three categories, the ordinate indicates the number of differential proteins under each functional classification. The color of the bar graph indicates the importance of rich GO function classification, which is the P value calculated based on Fisher's exact test. The label above the bar graph displays richFator (richFator $<=1$ ), which represents the proportion of the number of differentially expressed proteins annotated to a GO functional category to the number of all identified proteins annotated to the GO functional category. 


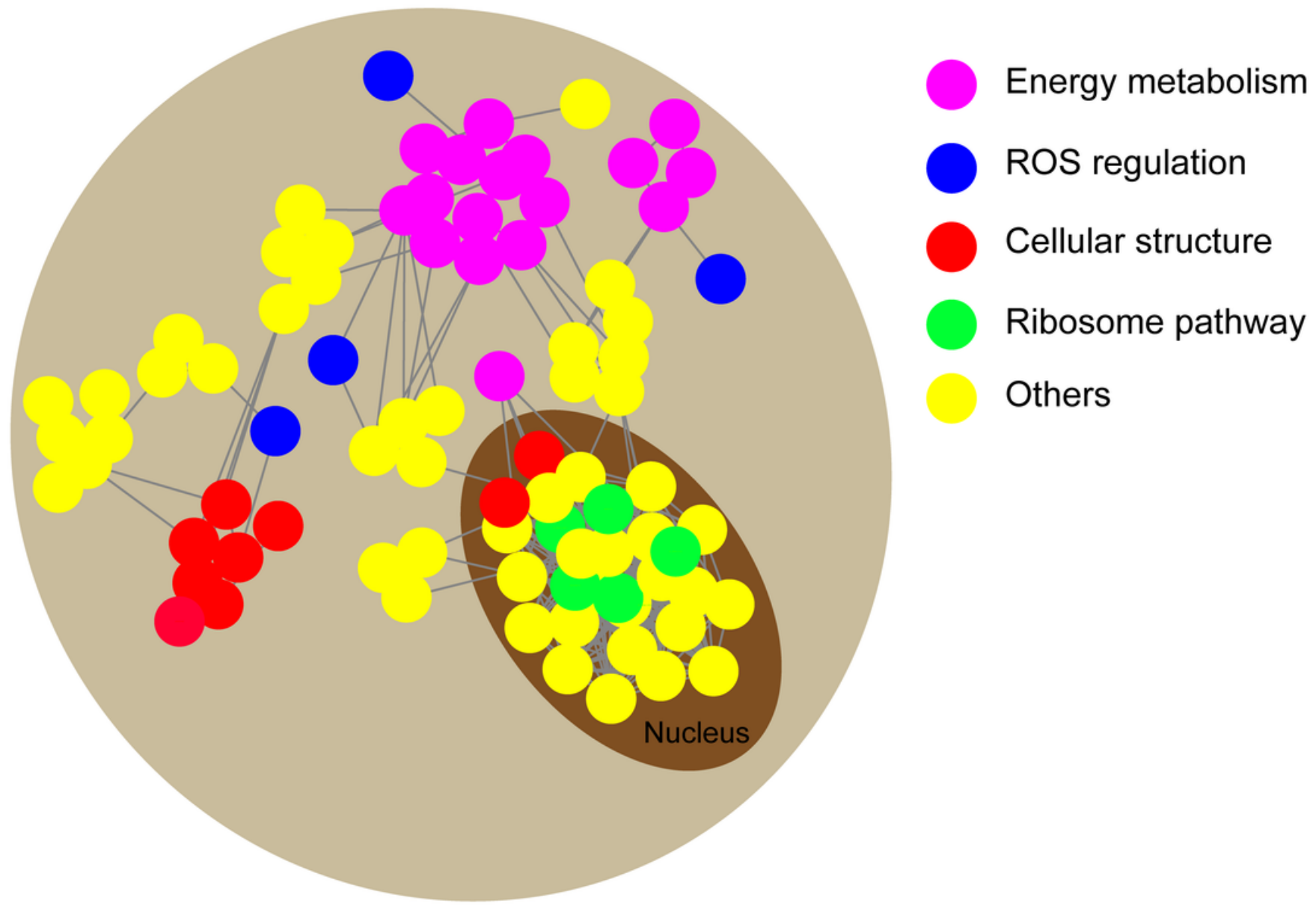

Figure 3

PPI network of differential protein under 17235T-17033T. The Purple cycle are energy metabolism proteins, blue cycle are ROS regulation proteins, red cycle are cellular structure proteins, green cycle are ribosome pathway proteins, yellow cycle are others proteins, and the protein in the dark brown ellipse is the protein predicted to be in the nucleus. 


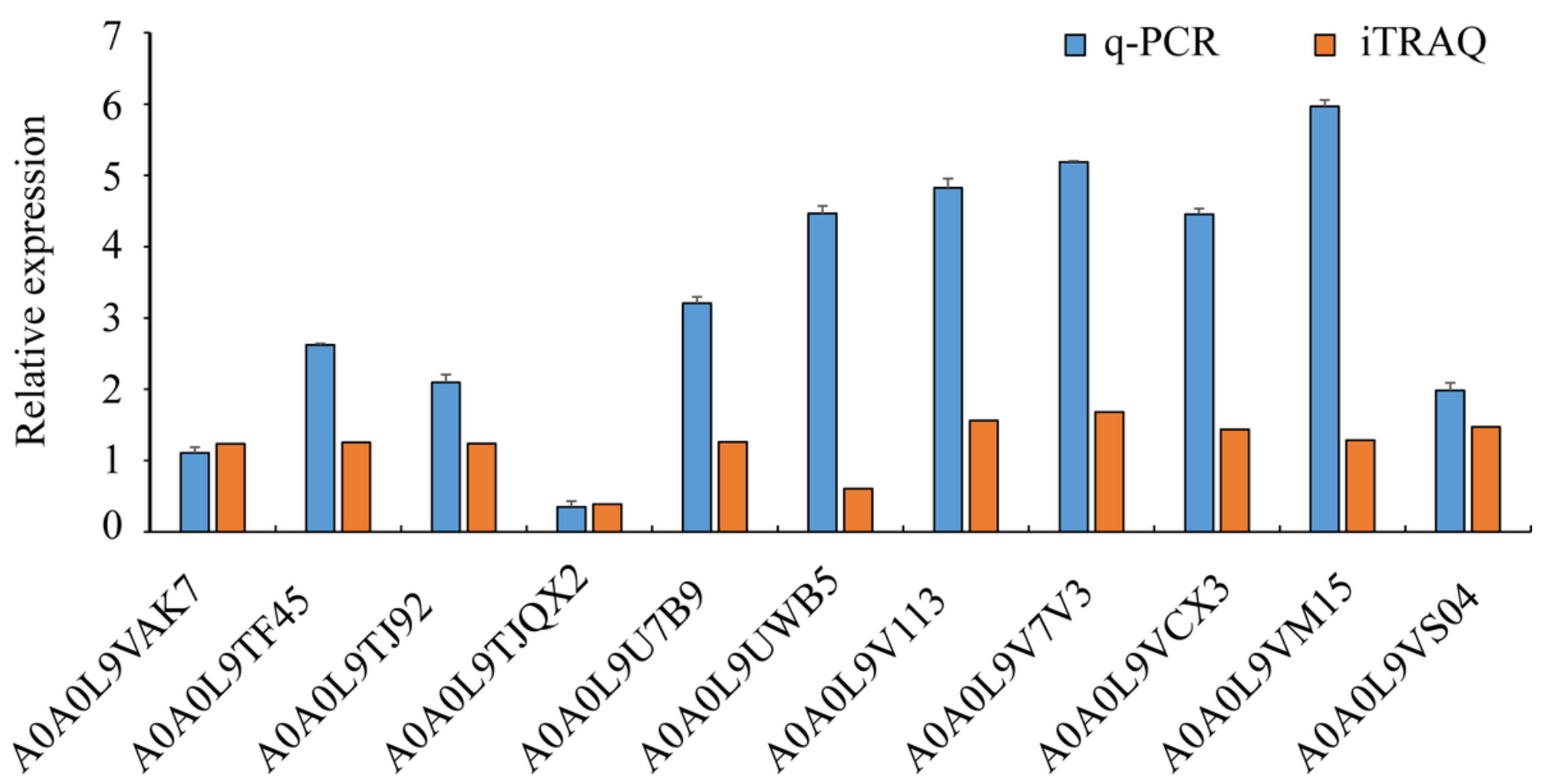

Figure 4

Differential protein quantification at transcript and translation level. Differences in protein expression and qRT-PCR between 17235 and 17033 under drought stress. q-PCR represents RNA expression level, iTRAQ represents the differences in protein expression level. 
A
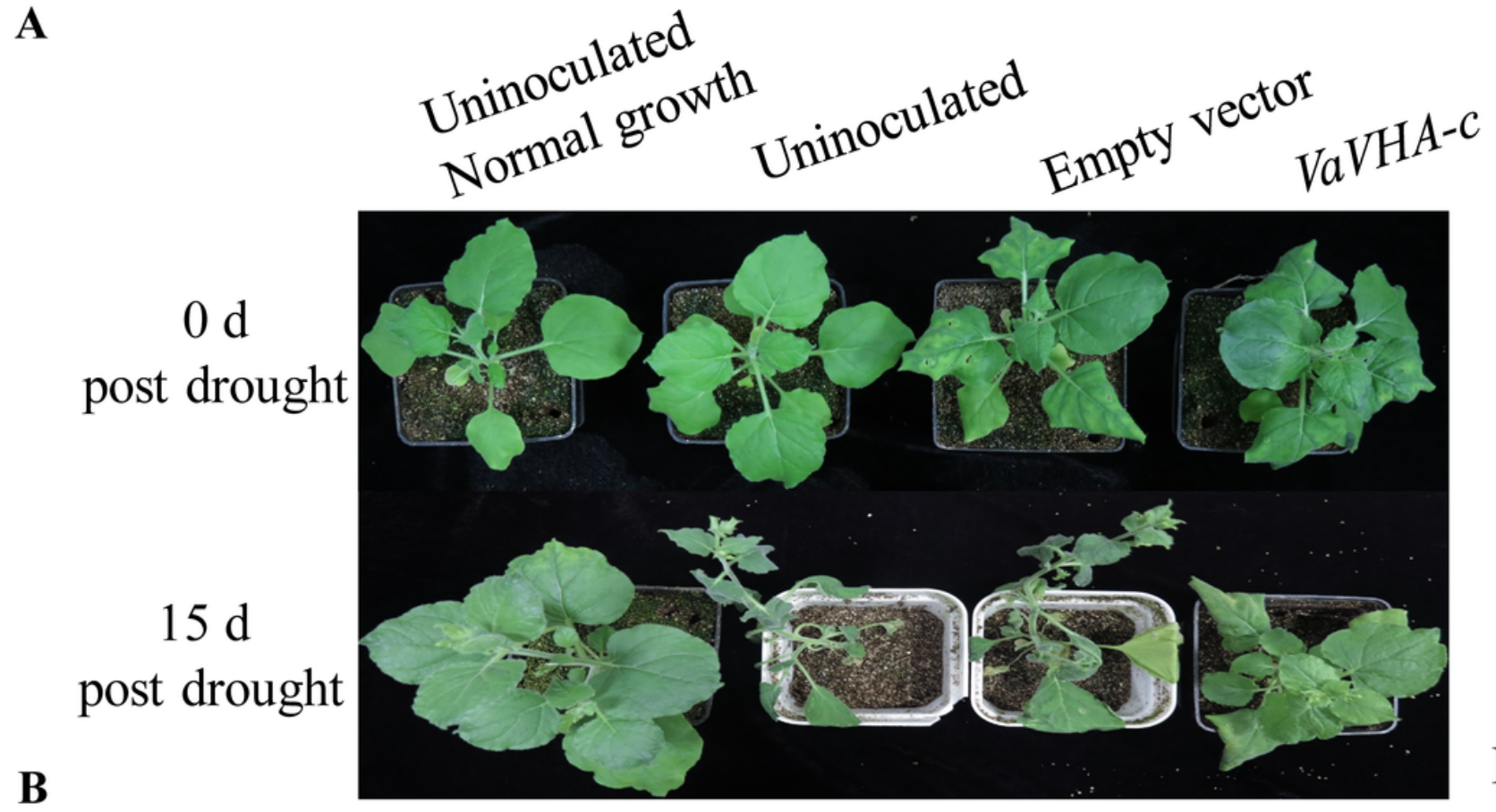

Maker

VaVHA-c

Actin

\section{Figure 5}

Function validation of VaVHA-c. A, phenotype of plants with overexpressed VaVHA-c, empty PVX vector, or uninoculated growth under normal condition ( $0 \mathrm{~d}$ post drought) or drought stress condition ( $15 \mathrm{~d}$ post drought). B, expression of VaVHA-c detected by RT-PCR. 
A

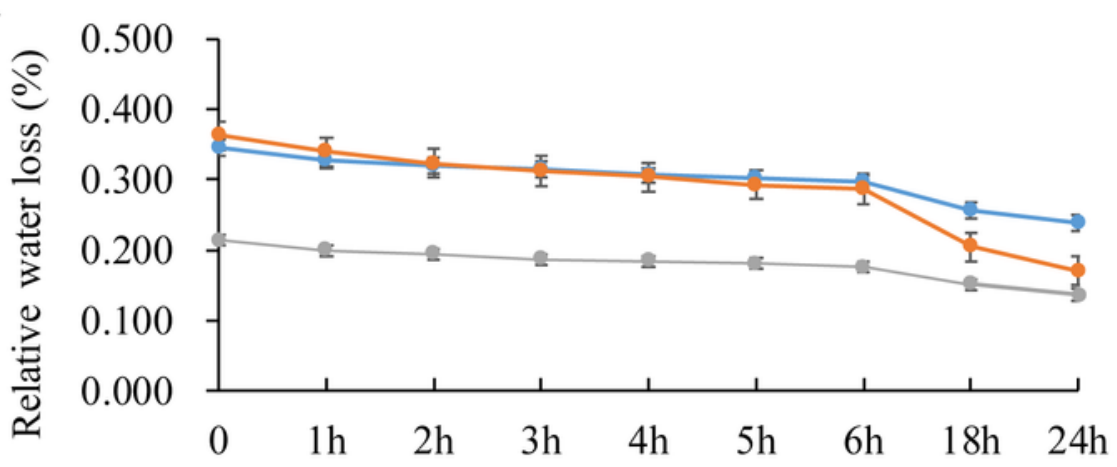

$\rightarrow$ Uninoculated

$\rightarrow$ Empty vector

$\rightarrow$ VaVHA-c

- Uninoculated

- Empty vector

a VaVHA-c
B

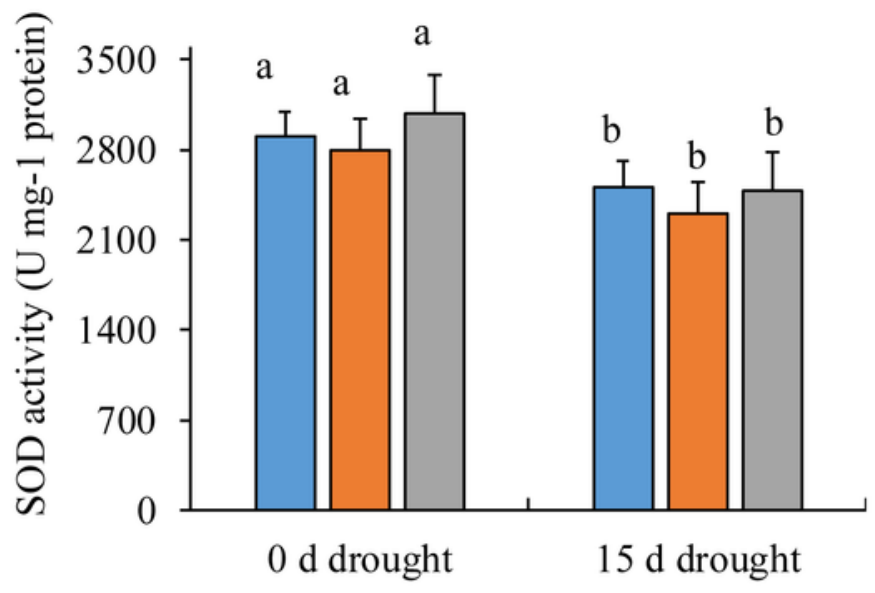

D

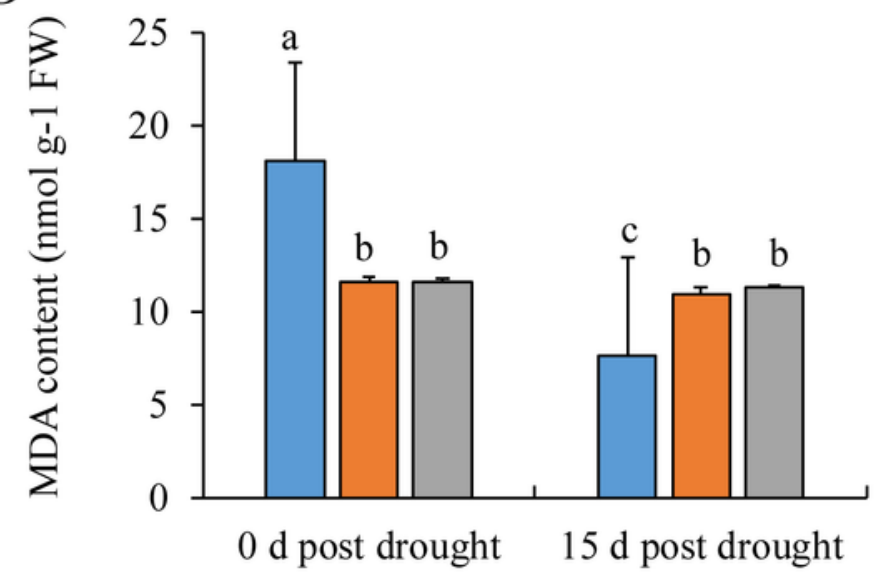

$\mathrm{C}$

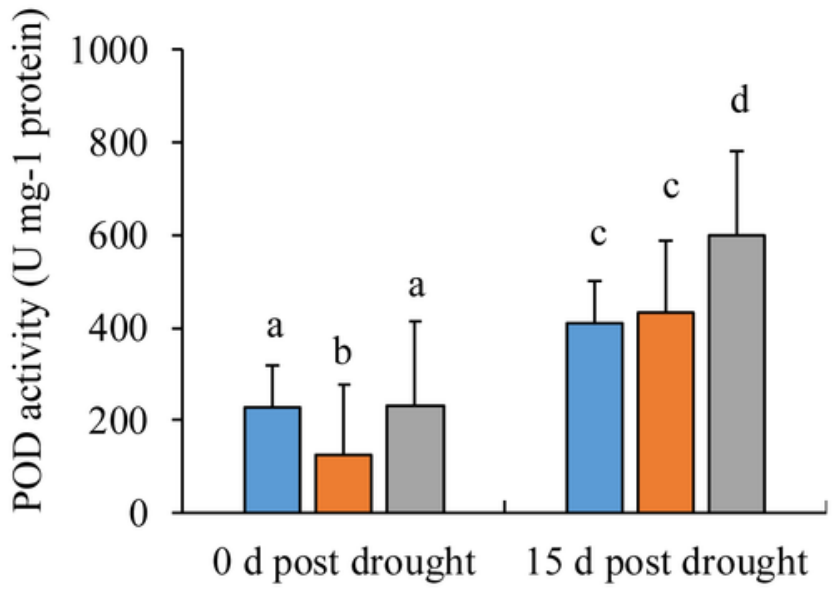

E

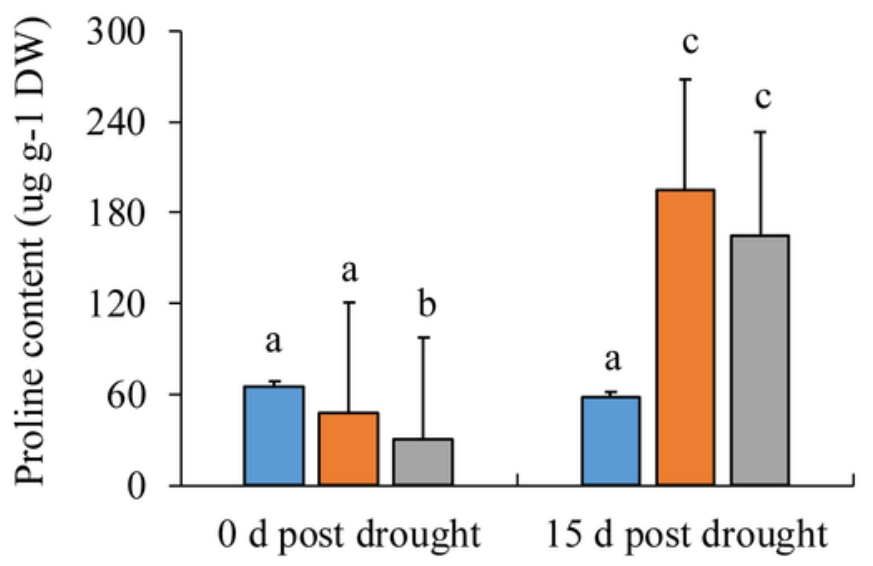

\section{Figure 6}

Physiological analysis of VaVHA-c ectopic expressed tobacco under drought. A, Water loss, B, C, Activity of SOD and POD, respectively, D, E, Content of MDA and proline, respectively. DW, dry weight, FW, Fresh weight, MDA, malondialdehyde, SOD: superoxide dismutase, POD: peroxidase. The lowercase letters represent significant difference $(P<0.05)$ between ectopic expressed lines and control plants. Values are means \pm SE of three replicates. 

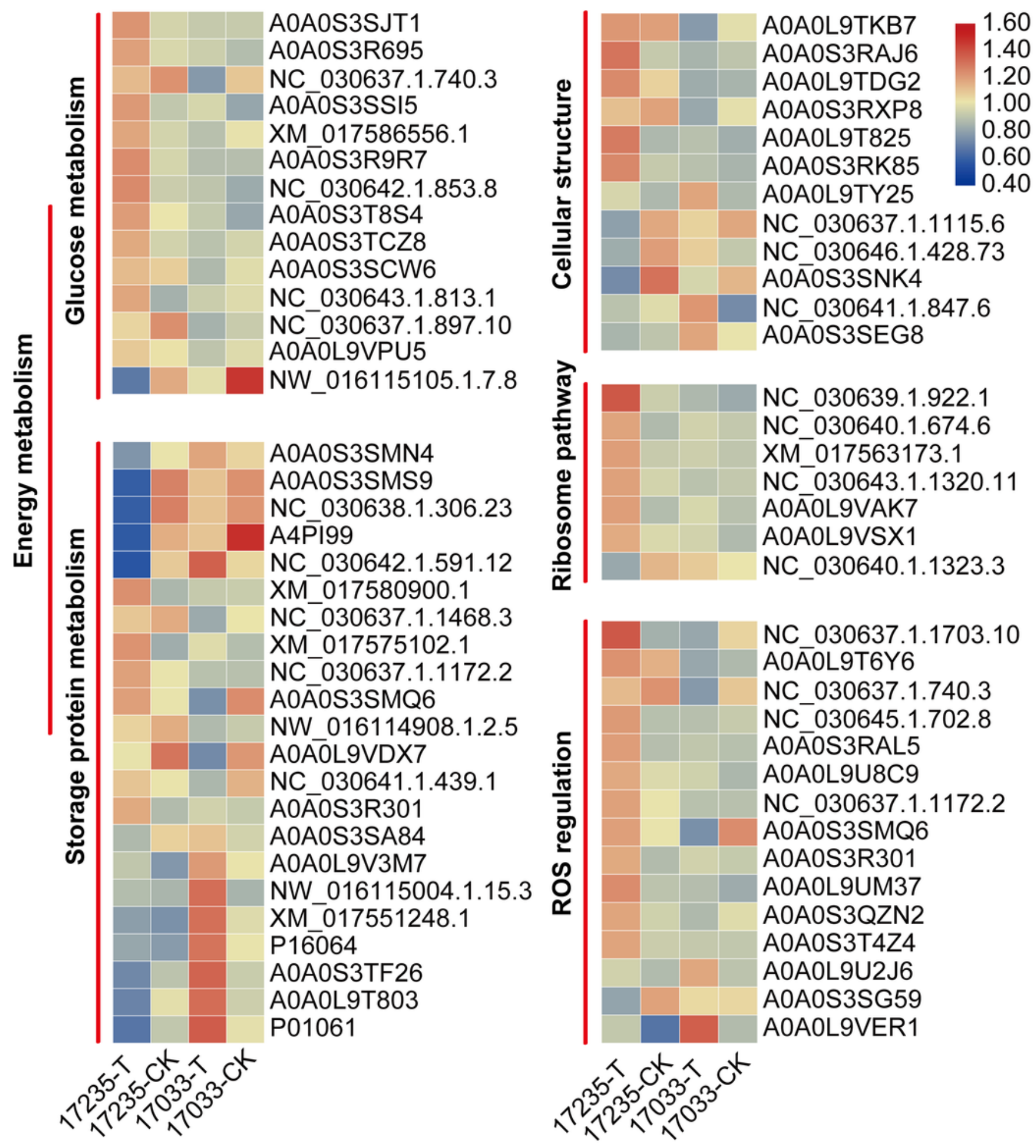

Figure 7

Heat map of four functionally grouped differential proteins.

\section{Supplementary Files}

This is a list of supplementary files associated with this preprint. Click to download. 
- Tables1.xlsx

- Tables2.xIsx

- TableS3.xlsx

- Tables4.xIsx

- Tables5.xlsx

- TableS6.xlsx

- Tables7.xIsx

- Tables8.xlsx 